\title{
Performance of a simulated-annealing algorithm for phase retrieval
}

\author{
M. Nieto-Vesperinas and R. Navarro \\ Instituto de Optica, Consejo Superior de Investigaciones Científicas, Serrano, 121, 28006 Madrid, Spain
}

\author{
F. J. Fuentes
}

Instituto de Astrofísica de Canarias, 38071 La Laguna, Tenerife, Spain

Received March 10, 1987; accepted June 10, 1987

\begin{abstract}
We report computer observations on the performance of an improved version of a simulated-annealing algorithm that was used before for the problem of phase retrieval. According to the results, we propose to use this method in conjunction with the algorithm of Fienup [Opt. Lett. 3, 27 (1978); Opt. Eng. 18, 529 (1979); Appl. Opt. 21, 2758 (1982). The full power of the simulated-annealing algorithm with large arrays appears to be limited by present-day computers rather than by its numerical performance, but we believe that this combination may constitute an efficient method for phase retrieval.
\end{abstract}

\section{INTRODUCTION}

The question of phase retrieval often appears in optics and other areas of physics such as $\mathrm{x}$-ray diffraction, scattering, and astronomy.

We shall deal with this subject for the case in which one intensity measurement is available in the Fourier domain. At present it is widely accepted that although phase reconstruction is nonunique in one dimension, there is a high probability that it is unique in two or more dimensions ${ }^{1,2}$ [although a certain amount of noise (uncertainty) in the data could increase the chance that they were approximately compatible with those of a nonunique situation].

Let $g(x, y)$ be a function representing a two-dimensional object with finite support (i.e., zero outside a certain domain), and let $G(u, v)=|G(u, v)| \exp [i \phi(u, v)]$ be its Fourier transform; the phase problem consists of finding the phase $\phi(u, v)$ from the known modulus $|G(u, v)|$ and certain restrictions on the object $g(x, y)$. Here, we shall consider the restrictions to be nonnegativity and finite support. In the $(x, y)$ space, with digital methods, assuming a discrete mod$\mathrm{el}$, this is equivalent to solving the set of nonlinear equations described by

$$
\begin{aligned}
Q_{i j}=\sum_{m=1}^{M} \sum_{n=1}^{M} g_{m n} g_{m+i-M, n+j-M}, & \\
1 & \leq m \leq M, \quad 1 \leq m+i-M \leq M, \\
1 & \leq n+j-M \leq M, \quad 1 \leq n \leq M,
\end{aligned}
$$

$g_{m n}$ being the $M^{2}$ samples (pixels) of the object function $g(x$, $y$ ) to be determined and $Q_{i j}$ representing the $(2 M-1)^{2}$ sampling data of the autocorrelation function $Q(x, y)$ of $g(x$, $y)$. Since $Q(x, y)$ is an even function, the equation set described by Eq. (1) contains, in fact, $M^{2}+(M-1)^{2}$ independent equations from which the $M^{2}$ unknowns $g_{m n}$ are to be found.
Among the several methods proposed so far to solve this problem, ${ }^{3-14}$ the most effective one is probably the combination of the error-reduction and the hybrid input-output versions of the iterative transform algorithm of Fienup. ${ }^{3-5}$ (We shall refer to this combined algorithm as FA from now on.) However, like other methods, this algorithm sometimes stagnates; i.e., the reconstruction becomes trapped in a local minimum of the corresponding cost function. Some procedures for overcoming three kinds of stagnation were reported quite recently. ${ }^{15,16}$ In fact, the number of local minima dramatically increases with the size of the object array ${ }^{14}$; this significantly affects Newton or steepest-descent methods.

In 1983 Kirkpatrick et al. ${ }^{17}$ put forward a Monte Carlo method called simulated annealing (SA) to avoid stagnation by nonzero minima of the cost function when solving large systems of nonlinear equations. This procedure is based on an analogy with the Monte Carlo method devised by $\mathrm{Me}-$ tropolis et $a l .{ }^{18}$ to calculate averages in statistical physics. SA has been used in optics for object reconstruction from coded images. ${ }^{19,20}$ Recently, some preliminary results with computer simulations of synthetic objects were reported ${ }^{21}$ that show the possibilities of SA in solving Eq. (1).

It should be emphasized that the convergence of SA is asymptotic; it never provides exact reconstructions in a finite number of iterations except for small object arrays. However, the solution that it yields to the phase problem may be significantly improved by increasing the number of iterations and also by repeating the algorithm several times. In all cases tested so far it has produced recognizable estimates of the true solution. Also, as will be shown, the algorithm is robust to noise and flexible; i.e., it permits improvements of the reconstruction by means of changes in its schedule. The main disadvantage of SA is that it is computationally costly. With present-day computers its use seems to be limited to object arrays of the order of $64 \times 64$ pixels at most. This fact was pointed out before, ${ }^{21}$ and it is well 
known to workers using SA in other areas of physics. Nevertheless, as we shall show, for objects of size, say, $32 \times 32$, this algorithm appears to be quite useful when FA stagnates (the actual tractable size of the object obviously depends on the computer at hand and on the time proposed to solve the problem).

In this paper we report a substantial improvement of the SA algorithm described in Ref. 21 for phase retrieval. First, its performance in yielding reliable reconstructions from Fourier modulus data has been improved by allowing the scale of pixel perturbation to vary according to a certain pattern. We have observed that this procedure is far superior to the quenching schedule discussed in Ref. 21. Second, its computational time has been reduced by a factor of approximately 6 with respect to the version of Ref. 21 so that now an object reconstruction of $32 \times 32$ pixels costs about 12 $h$ of central processing unit (CPU) time in a Data General or VAX computer and less than $3 \mathrm{~h}$ in a CDC-Cyber. These times are still considerably higher than those of FA (about 15 min in a Data General or VAX computer for a $32 \times 32$ pixel object if it is successful, although it will require further trials if it stagnates). However, we believe that SA is a reliable and helpful algorithm to be used if FA stagnates, yielding a good estimate of the solution in all cases, as will be shown in the following sections. Therefore the aim of this paper is to show the performance of SA in those cases in which the FA reconstruction is not satisfactory. One may then take advantage of the speed of FA by introducing its reconstruction as a first input of the SA algorithm. In fact, the SA algorithm can give a solution independently of the use of FA and of the input guess, but the profit of FA output in SA can provide a shortcut saving, in most cases, of a considerable amount of computing time. In any case, we propose first to use FA and then to introduce the SA algorithm to improve the reconstruction when it is necessary.

In Section 2 we shall briefly review the essentials of the SA method for phase retrieval. In Section 3 we shall compare its performance with that of FA and show its capability for improving FA reconstructions. First it will be stated that FA can provide almost exact reconstructions from noiseless data when it works in a comparatively short computing time. However, FA does not exhibit a uniform behavior with different objects; sometimes it stagnates. ${ }^{15,16}$ This will be seen with two astronomical objects of large dynamic range. On the other hand, the SA algorithm has features that are complementary with respect to FA. SA provides an exact reconstruction only asymptotically, even from noiseless data. However, we have observed a uniform behavior of SA with different objects, and the quality of the reconstructions showed in Section 3 increases with the computing time, namely, with the number of steps involved in the SA process.

\section{A REVIEW OF THE SIMULATED- ANNEALING ALGORITHM}

The solution to the system of equations described by Eq. (1) is sought by finding the global (zero) minimum of the cost function,

$$
F\left(g_{m n}\right)=\left[\sum_{k=1}^{M^{2}+(M-1)^{2}} r_{j k}^{2}\right]^{1 / 2}, \quad k=M(i-1)+j
$$

where the residuals $r_{k}$ are

$$
\begin{aligned}
r_{k}=Q_{i j}-\sum_{m=1}^{M} \sum_{n=1}^{M} g_{m n} g_{m+i-M, n+j-M}, & 1 \leq m+i-M \leq M, \\
1 \leq n+j-M \leq M, & k=M(i-1)+j .
\end{aligned}
$$

In practice, for noisy data some $k$ th residuals $r_{k}$ corresponding to pixels near the borders of the autocorrelation function may be multiplied by a parameter $\lambda$ that is equal to zero if

$$
\sum_{m=1}^{M} \sum_{m=1}^{M} g_{m n} g_{m+i-M, n+j-M}
$$

is less than a threshold value. (We have set it at $10^{-4}$ times the maximum value of the autocorrelation array $Q_{i j}$. This is also the approximate order of the values of the pixels $Q_{i j}$ near the borders.) On the other hand, $\lambda$ is made greater than 1 (we have used $\lambda=5$ ) if

$$
\sum_{m=1}^{M} \sum_{m=1}^{M} g_{m n} g_{m+i-M, n+j-M}
$$

is greater than the above threshold. In this way, those pixels near the borders of the autocorrelation array of the reconstruction that have values of the same order as the noise level will gain weight if they differ from the data.

Another way of giving more weight to the autocorrelation pixels far away from the center of the autocorrelation array is to scale Eq. (1), for instance, by dividing each $r_{k}$ by the number of terms contributing to the corresponding $Q_{i j}[k=$ $M(i-1)+j]$. Evidently, this number is $M^{2}$ for the residual corresponding to the central pixel and is 1 for those in the outer corners.

The SA method is based on a theorem stating that if $F(\mathbf{x})$ is an $N$-dimensional well-behaved function with a global minimum $x^{*}$ in a domain $S$ of $R^{N}$, then ${ }^{22-24}$

$$
\mathbf{x}^{*}=\lim _{T \rightarrow 0} \frac{\int_{s} \mathrm{~d} \mathbf{x} \mathbf{x} \exp [-F(\mathbf{x}) / T]}{\int_{s} \mathrm{~d} \mathbf{x} \exp [-F(\mathbf{x}) / T]} .
$$

$\mathbf{x}^{*}$ is then obtained by calculating the averages of $\mathbf{x}$ with a probability density $\exp [-F(\mathrm{x}) / T]$ at each $T$ by means of a Monte Carlo method put forward by Metropolis et al. ${ }^{18}$ This is done by generating at each fixed $T$ an irreducible Markov chain with a probability $\exp [-F(\mathbf{x}) / T]$, such that in the long run the transition probabilities that increase $F(\mathbf{x})$ equal those that decrease $F(\mathbf{x})$; i.e., at that stage the configuration of $\mathbf{x}$ remains at equilibrium. The global minimum $\mathbf{x}^{*}$ is then the limit as $T \rightarrow 0$ of the successive equilibrium configurations of $\mathbf{x}$ obtained at each $T$. More details on the mathematical formulations of this method may be found in Refs. 17, 18, and 22-24.

The procedure is performed as follows: one starts with an initial guess object $\left\{g_{m n}{ }^{(0)}\right\}$ whose corresponding cost function will be $F^{(0)}$, one fixes the value of $T$, and then one perturbs the first pixel $g_{00}$ of $\left\{g_{m n}{ }^{(0)}\right\}$ by a certain amount $h$ chosen from a sequence of computer-generated random numbers uniformly distributed in the interval $(-\alpha, \alpha) ; \alpha$ will be called the scale of perturbation. The cost function $F^{(1)}$ of the new object is evaluated. One then computes the difference: 


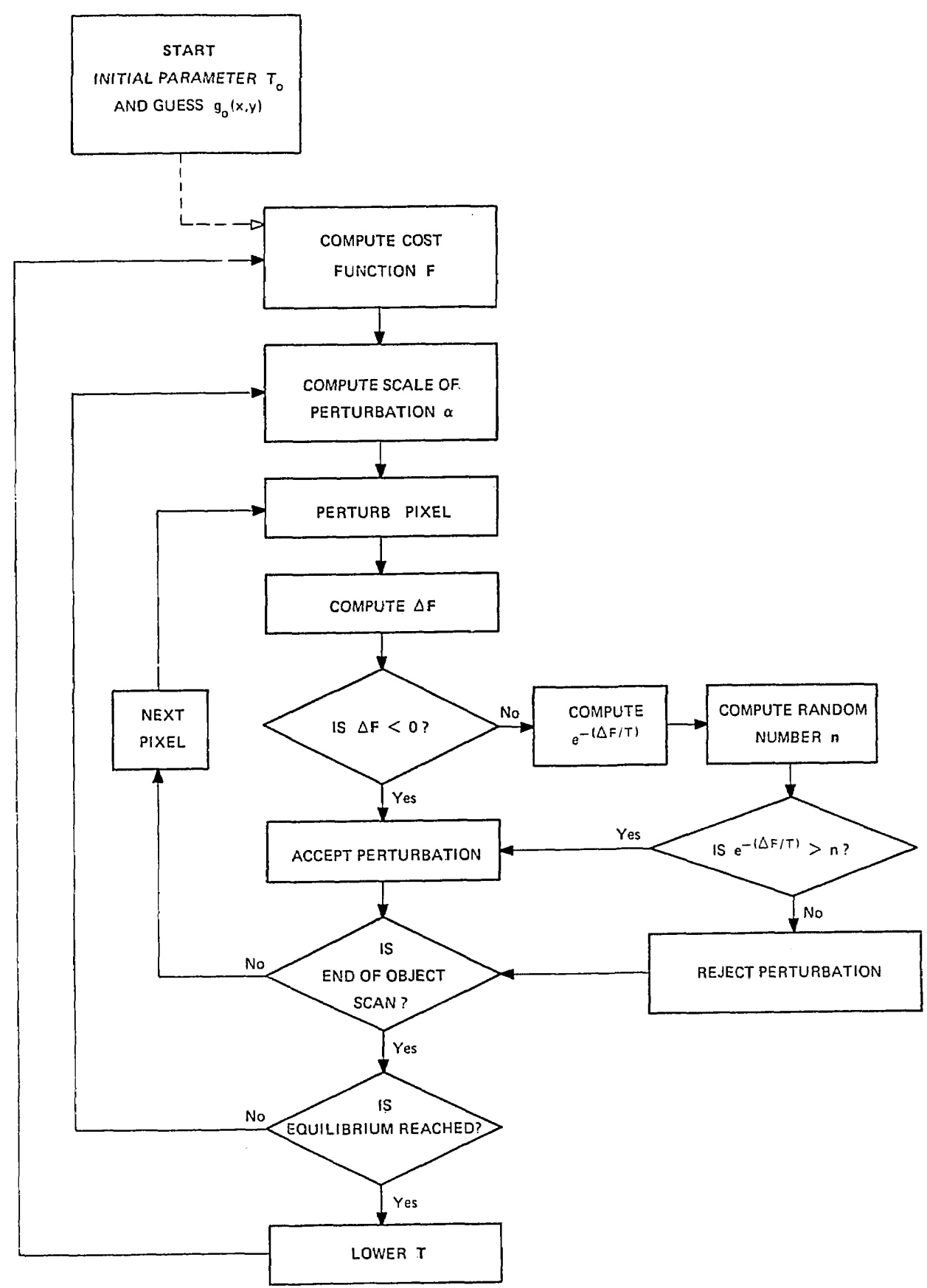

Fig. 1. Block diagram of the SA algorithm.

Plate 1. Scale associating colors with object intensities.

Plate 2. (a) Original galaxy object compressed to $32 \times 32$ pixels. (b) FA reconstruction from noisy data with a $\mathrm{S} / \mathrm{N}$ ratio of 10 . (c) Low-passfiltered version of (b). (d) SA reconstruction with a $\mathrm{S} / \mathrm{N}$ ratio of 10 . (e) Low-pass-filtered version of (d). (f) FA reconstruction with a S/N ratio of 4. (g) SA reconstruction with a S/N ratio of 4 .

Plate 3. (a) FA reconstruction from simulated speckle interferometry data. (b) Low-pass-filtered version of (a). (c) SA reconstruction from simulated speckle interferometry data. (d) Low-pass-filtered version of (c). (e) Reconstruction combining FA and the SA algorithm from simulated speckle interferometry data. (f) Low-pass-filtered version of (e).

Plate 4. (a) Original two-nucleus quasar object compressed to $32 \times 32$ pixels. (b) FA reconstruction from noiseless data. (c) Reconstruction from noiseless data combining FA and the SA algorithm.

Plate 5. (a) FA reconstruction of the object shown in Plate 2(a) from noiseless data. (b) Reconstruction from noiseless data by combining FA and 10 repetitions of the $\mathrm{SA}$ algorithm. 


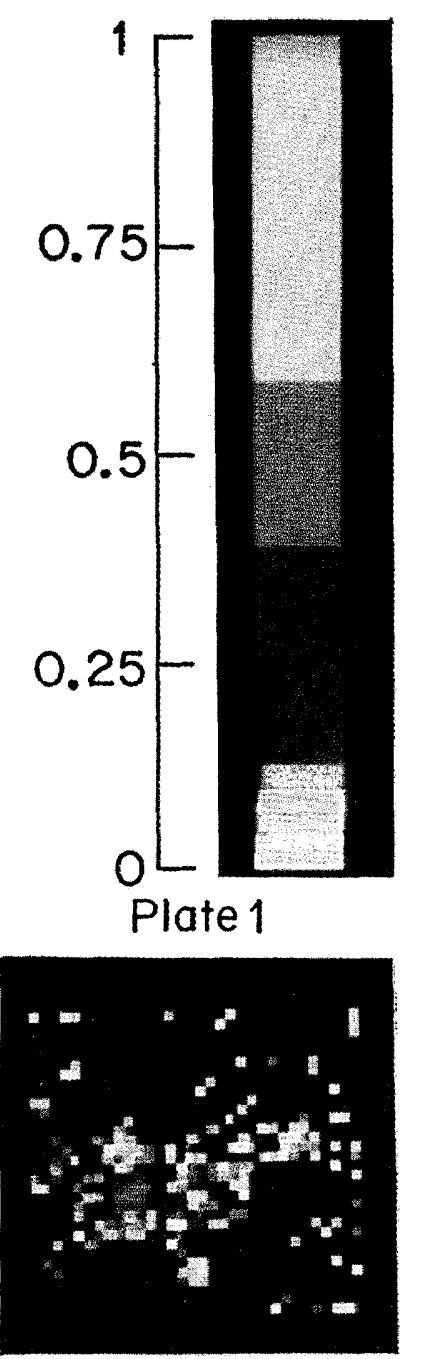

Plate 2(g)

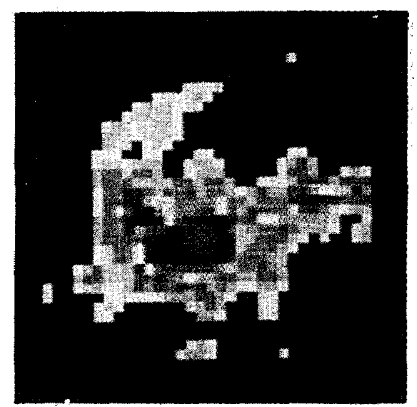

Plate 3(d)

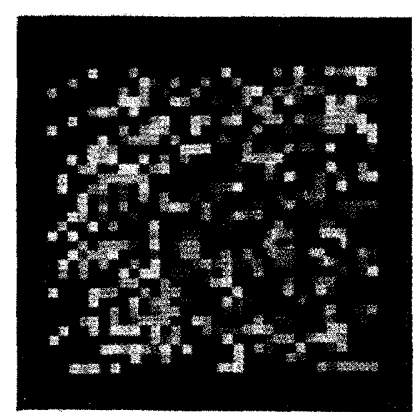

Plate 4(b)

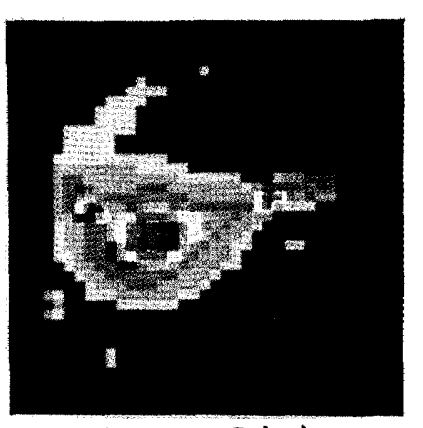

Plate 2(a)

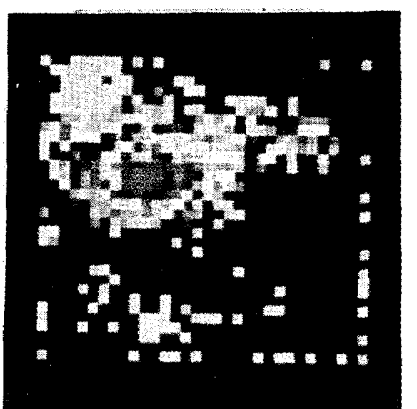

Plate 2(d)

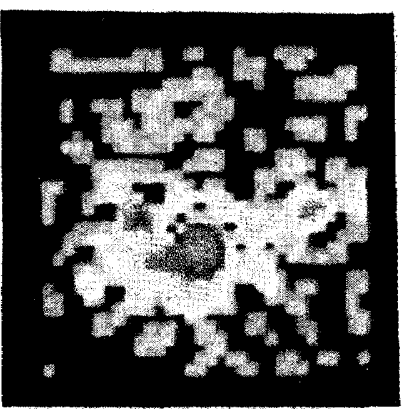

Plate 3(a)

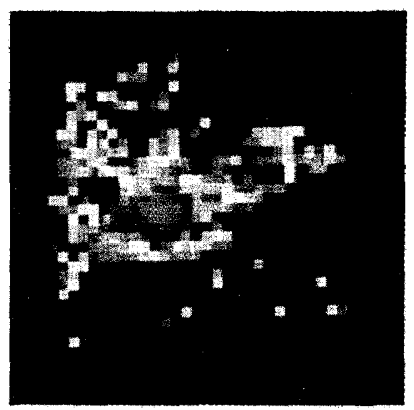

Plate 3(e)

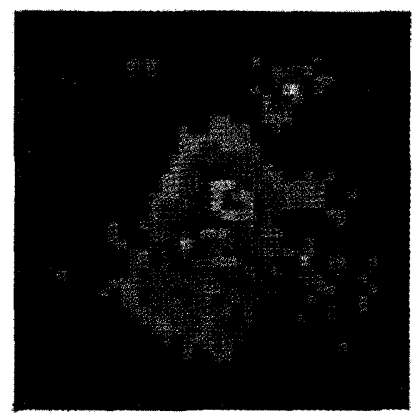

Plate 4(c)

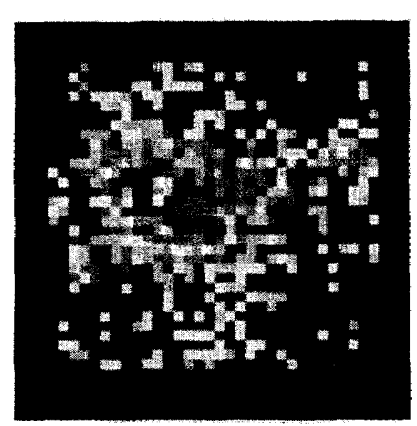

Plate 2(b)

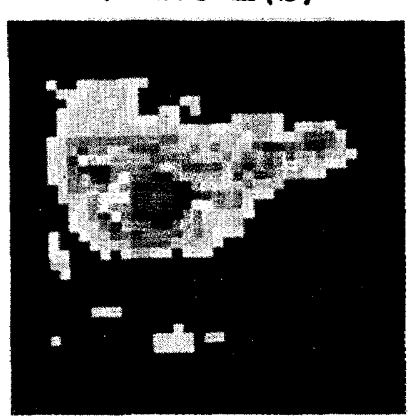

Plate 2(e)

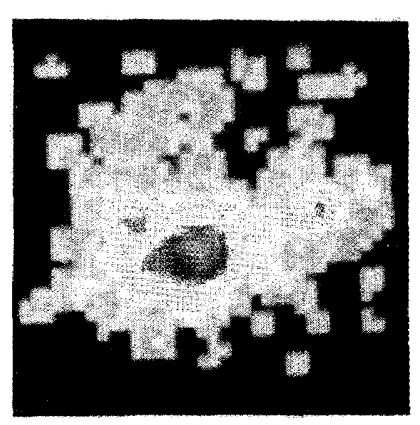

Plate 3(b)

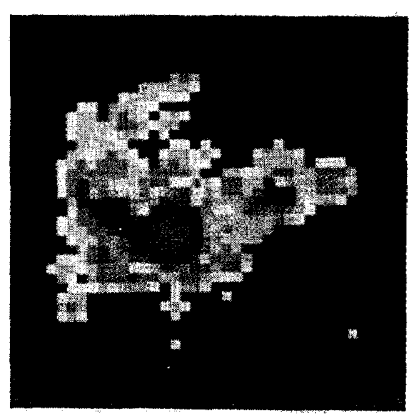

Plate 3(f)

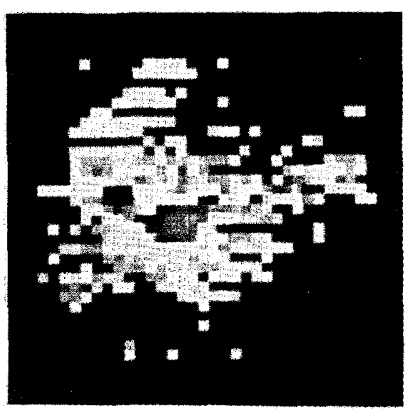

Plate 5(a)

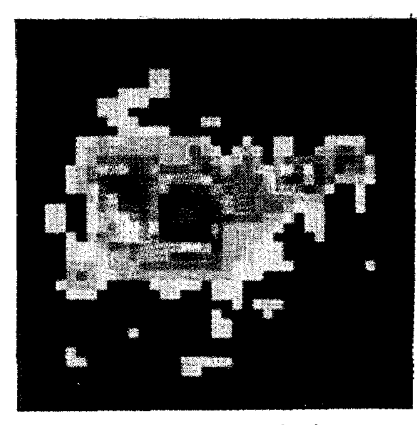

Plate 2(c)

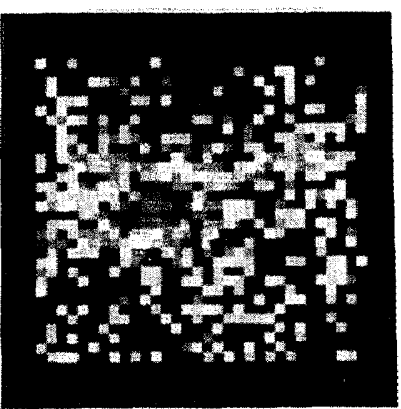

Plate 2(f)

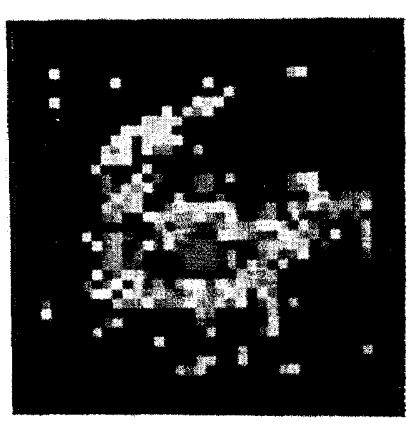

Plate 3(c)

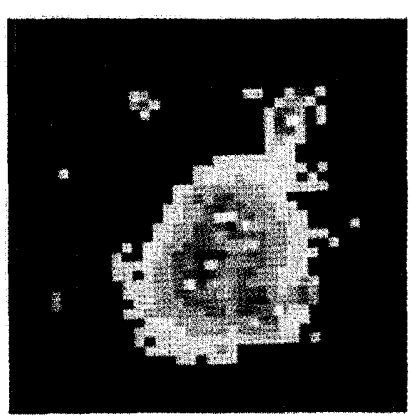

Plate 4(a)

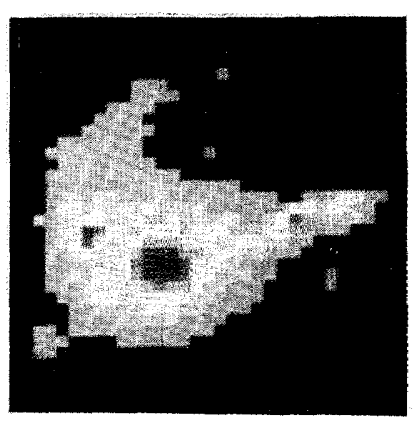

Plate 5(b) 



$$
\Delta F=F^{(1)}-F^{(0)} .
$$

If $\Delta F \leq 0$, then the change is accepted, and one passes to the next pixel; if $\Delta F>0$, then the change is accepted with a probability $\exp (-\Delta F / T)$. To do this, we take a number $\mathbf{n}$ from a sequence of random numbers uniformly distributed in the interval $(0,1)$. If $\mathbf{n}$ is less than $\exp (-\Delta F / T)$, then the change is accepted, and one starts with the next pixel. If $\mathbf{n}$ is greater than or equal to $\exp (-\Delta F / T)$, then the change is not accepted, and one starts with the next pixel. All pixels of the object are scanned in this way for as many cycles as necessary until equilibrium is reached, i.e., when on the average the number of trials increasing $F$ equals the number of trials decreasing $F$. $T$ is then lowered, and one starts the procedure again. The larger $T$ is, the higher the probability is of accepting a change raising $F$. Thus, since $F$ is allowed to increase, one is not likely to get stuck in the nearest local minimum. Eventually, when one is near the end of the algorithm, at very low values of $T$, only changes lowering $F$ are accepted.

The scale of perturbation $\alpha$ must be chosen with care; if $\alpha$ is too large, $F$ will oscillate highly or will yield forbidden changes, and if $\alpha$ is too small, the reconstruction evolution will be too slow. The performance of the algorithm is enhanced if $\alpha$ is allowed to decrease as $T \rightarrow 0$ according to a certain schedule.

The fast computation of the autocorrelation function of the corresponding iteration with a perturbed pixel is a nontrivial matter. The most efficient way that we have found is to follow the scheme

$$
\begin{aligned}
Q^{(m+1)}(x, y)= & \iint \mathrm{d} \xi \mathrm{d} \eta\left[g^{(m)}(\xi, \eta)+h \delta(\xi-a, \eta-b)\right] \\
& \times\left[g^{(m)}(\eta+x, \eta+y)+h \delta(\xi+x-a, \eta+y-b)\right] \\
= & Q^{(m)}(x, y)+h g^{(m)}(a-x, b-y) \\
& +h g^{(m)}(a+x, b+y)+h^{2} \delta(x, y)
\end{aligned}
$$

In Eq. (6), $Q^{(m+1)}(x, y)$ is the autocorrelation function resulting from adding an amount $h(-\alpha \leq h \alpha)$ to the pixel in $(a, b)$ of the $m$ th iteration of the object $g^{(m)}(x, y)$, and $\delta(x, y)$ is the $\delta$ function.

A FORTRAN routine for the whole algorithm will be reported soon. ${ }^{25}$ A block diagram of the algorithm is shown in Fig. 1.

\section{COMPUTATIONAL RESULTS}

Both the FA and the SA reconstructions were tested with 32 $\times 32$ pixel objects. The FA was done by embedding the object in a $64 \times 64$ pixel window array to perform fastFourier-transform operations. In all cases shown in this work the starting guess for both FA and SA was the one suggested by Fienup ${ }^{5}$ : take the autocorrelation data array $Q_{i j}$, save every other pixel in both dimensions and threshold it to a value (we used $10^{-4}$ with a maximum normalized to 1 ), and fill the results with random numbers. We usually obtain better performance of the algorithms by using this strategy than by starting with $32 \times 32$ pixel input arrays with random or constant values.

FA was used with a feedback parameter $\beta=0.7$ in the hybrid input-output parts. Twenty error-reduction inter- actions were done first; then we employed seven cycles, each containing one hundred hybrid input-output iterations followed by twenty error-reduction iterations.

In all cases tested in this work, no further evolution of the reconstruction was observed by considerably increasing this number of cycles above seven or ten.

In fact, $10 \mathrm{~h}$ of CPU time in a Data General computer and $2 \mathrm{~h}$ in a CDC Cyber computer were given to FA for a reconstruction from noiseless data of the objects of Plates 2(a) and 4(a). Neither reconstruction evolved from that of Plates 4(b) and 5(a), obtained with $28 \mathrm{~min}$ of CPU of FA, and the cost functions did not drop any further. This may be because of effects involved in the Fourier-transform operation, namely, the noisy and stripped features of Plates $4(\mathrm{~b})$ and 5 (a), respectively, seem to be due to biased phases acquired in the spectrum in the first iterations. No attempts were made to overcome stagnation by the methods of Refs. 15 and 16 , however.

For the SA algorithm, two annealing schedules were tested. One schedule features exponential decay, or $T$ as $T_{n}=$ $\exp [-k(n-1)] T_{0}$ in 50 steps $n . k$ is chosen such that $T_{0}=$ 100 and $T_{50} \simeq 10^{-6}$; i.e., $k=0.37$. The maximum number of cycles of object scan is set equal to 100 . Equilibrium is considered reached when three consecutives times at the end of a cycle the number of accepted perturbations raising $F$ differ from the number of perturbations lowering $F$ (always accepted) to within $5 \%$. The scale of perturbation $\alpha$ starts at $\alpha \simeq 1$ and is modified at the end of each cycle according to the schedule

$$
\alpha_{n}=B\left(\frac{A+\log F_{n}}{C}\right)^{P} .
$$

$A$ equals the order of the initial cost function $F_{0}\left(F_{0} \simeq 10^{5}\right.$; thus $A=5$ ). The exponent $P$ establishes the change rate of $\alpha(P=5$ worked well $), C$ is a number of chosen to make the quotient no greater than 1 (we used $C \simeq 16$ ), and $B$ scales the initial value $\alpha_{0}$ to approximately 1 (we set $B=10$ ).

The other schedule tested, which produced similar results, contained the same number of steps of $T$ as the first schedule but featured a linear decay of $T$; i.e., $T_{n+1}=0.75 T_{n}$ and $\alpha_{m}=$ $\exp [-(m-1)]$, with the index $m$ increasing one unit every seven steps of variation in $T$.

The chosen schedule therefore depends largely on the user. In any case, these choices of $T$ and the scale of perturbation $\alpha$ are by no means exhaustive and must be considered merely concrete examples of performance of the SA algorithm in our case. A general rule, however, may be established, namely, that both the decay in $T$ and the decay in $\alpha$ must be performed carefully and slowly. Otherwise, one is likely to be trapped in a local minimum of the cost function.

All the examples to be shown in what follows took about 15-30 min of CPU time for FA and about 12-14 h of CPU time for SA in a Data General Eclipse MV 10000 computer. However, when the SA reconstruction was started with the FA result, the CPU time was reduced to about $6 \mathrm{~h}$, provided that one tried to save perturbation scans near equilibrium. As mentioned before, with the astronomical objects, no improvement of the reconstructions was observed in the examples by letting FA run over comparable computing times.

Since there are discussions among users on their relative successes in applying FA, we wish to report first a remarkable performance of this algorithm with a difficult synthetic 


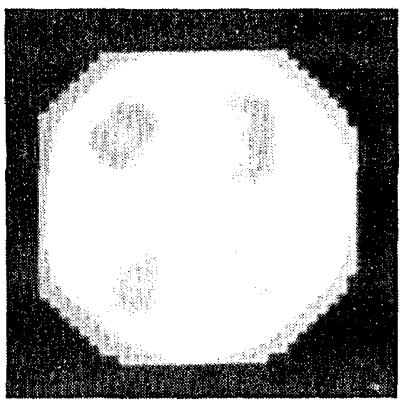

(a)

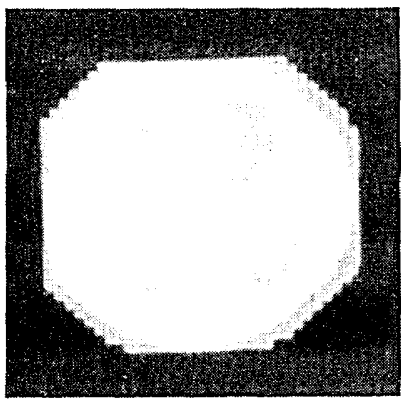

(b)
Fig. 2. (a) Original object, which represents a $32 \times 32$ pixel lowpass-filtered version of object 5 of Ref. 21. (b) FA reconstruction from noiseless data.

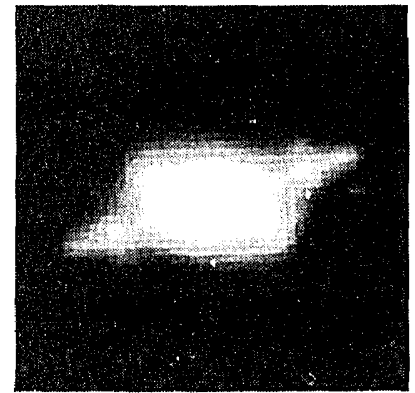

(a)

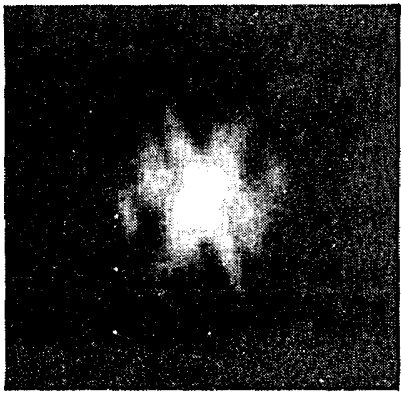

(b)
Fig. 3. (a) Autocorrelation and (b) power spectrum of the object shown in Plate 1(a).

object such as the one shown in Fig. 5 of Ref. 21 (excluding one file and one column to make it $32 \times 32$ pixels). Fienup's error function [Eq. (4) of Ref. 3] for the initial guess was $E_{0}=$ 0.22. After FA was applied to noiseless data, the reconstructed object showed an error of $E=2.3 \times 10^{-4}$, and its visual appearance was indistinguishable from the original. The SA version reported here, although it is a considerable improvement of the result in Ref. 21 , yielded a poorer reconstruction, with $E \simeq 10^{-3}$. It should also be remarked that no additional a priori information or constraint was used. FA performance was very satisfactory even with a signal-tonoise $(\mathrm{S} / \mathrm{N})$ ratio of 10 in the spectrum data, yielding a reconstruction with $E=2.6 \times 10^{-2}$. The $\mathrm{S} / \mathrm{N}$ ratio used here was

$$
\mathrm{S} / \mathrm{N} \text { ratio }=\frac{\int|G(u, v)| \mathrm{d} u \mathrm{~d} v / \mathcal{A}}{\sigma},
$$

where $\mathcal{A}$ is the power spectrum area, $\left(64^{2}\right.$ in our case) and $\sigma$ is the root mean square of the noise.

Similar satisfactory results were obtained with FA in reconstructions of low-pass-filtered versions of the same object showing much lower contrast. Figure 2(a) shows the original object, and Fig. 2(b) shows the reconstruction from noiseless data, with an error $E \simeq 10^{-4}$. In fact, no local minima of the kind reported in Fig. 2 of Ref. 26 were found, even in the low-pass-filtered object. Therefore, with this kind of object, FA appears to be a highly efficient method of phase retrieval.

However, the SA algorithm may be very helpful with objects of large dynamic range. The color scale for the plates printed in color is shown in Plate 1. Plate 2(a) shows a $32 \times 32$ pixel image of a galaxy recorded by astronomers of the Instituto Astrofisico de Canarias (IAC) with a charge-coupleddevice camera. We present it in color because of its large dynamic range. The autocorrelation and the power spectrum of this object are shown in Figs. 3(a) and 3(b), respectively. A logarithmic scale of gray levels has been used in Figs. 3(a) and 3(b); even so, the fainter outer details are not visible.

FA reconstruction of this object from noisy data in the power spectrum with a $\mathrm{S} / \mathrm{N}$ ratio of 10 is shown in Plate 2(b); the error is $E=1.7 \times 10^{-2}$. The error of the initial guess was $E_{0}=0.53$. In terms of the cost function of Eq. (2), the reconstruction of Plate $2(\mathrm{~b})$ has $F=10^{-2}$. The initial guess had a cost function $F_{0}=69922$. In Plate 2(c) a low-passfiltered version of Plate $2(\mathrm{~b})$ is shown. The visual appearance is less noisy, and the higher-density details are clearer, although the error and cost functions increase 1 order of magnitude. The SA reconstruction is shown in Plate 2(d); it has $E=3.2 \times 10^{-2}$ and $F=4.9 \times 10^{-2}$. A low-pass-filtered version of the same reconstruction is shown in Plate 2(e). These SA reconstructions are less noisy than those provided by FA, although they are shifted upward, which is probably the reason for their slightly higher error.

Plate 2(f) shows the FA reconstruction of the same object with a S/N ratio of 4 and with $E=4.4 \times 10^{-2}$ and $F=6.6 \times$ $10^{-2}$. On the other hand, plate $1(\mathrm{~g})$ shows the $\mathrm{SA}$ reconstruction from these data with $E=8.86 \times 10^{-2}$ and $F=9.1 \times$ $10^{-2}$. Once again, these errors are slightly higher than those of the FA reconstruction although its visual appearance is less noisy. Thus, although these cost and error functions are a good measure of the convergence of the corresponding algorithm, they have a certain discrepancy with respect to the visual quality of the reconstructions. Curiously, if one calculates the cost function of the above reconstructions, as in Eq. (2), but also divides each residual $r_{k}$ by the central maximum of the autocorrelation data, one obtains $F=2.5 \times$ $10^{-6}$ for the FA reconstruction in Plate $2(\mathrm{~b})$ and $F=1.6 \times$ $10^{-6}$ for the SA reconstruction in Plate 2(d) as well as $F=2.5$ $\times 10^{-5}$ for $\mathrm{FA}$ reconstruction in Plate 2 (f) and $F=1.18 \times$ $10^{-5}$ for the SA reconstruction in Plate $2(\mathrm{~g})$.

Plates 3(a)-3(f) show reconstructions from data obtained by means of a computer simulation of speckle interferometry data obtained by convolving the original object [shown in Plate 2(a)] with each of 100 speckled point-spread functions representing different realizations of the turbulent atmosphere according to a certain model. ${ }^{27}$ The resulting power spectrum data are practically equal to those of the noiseless

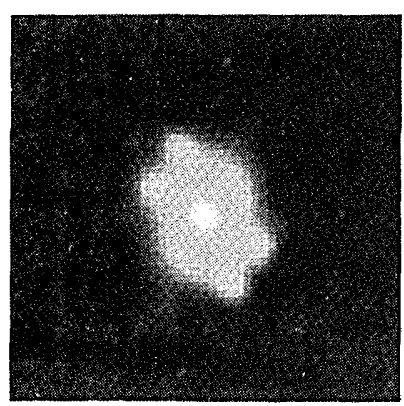

(a)

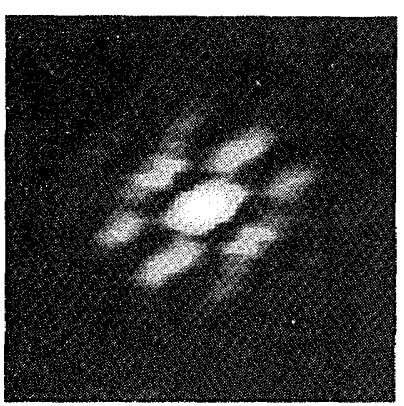

(b)
Fig. 4. (a) Autocorrelation and (b) power spectrum of the object shown in Plate 2(a). 
spectrum but are set to zero at high frequencies whenever they are less than a threshold value equal to $10^{-2}$ times the maximum. Plate $3(\mathrm{a})$ shows the reconstruction given by FA, and plate 3(b) shows a low-pass-filtered image of that picture. The reconstruction obtained by means of SA and its low-pass-filtered version are shown in Plates $3(\mathrm{c})$ and $3(\mathrm{~d})$, respectively. These are clearly less noisy than those of Plates 3(a) and 3(b).

Interesting results may be obtained by using a combination of FA and SA algorithms. Since FA is fast, one can take advantage of this by using it first; one can then attempt to improve the result by introducing it as a first guess in the SA algorithm. In this case the starting value of $T$, however, may be set much lower (according to the cost function).

In the example to be shown next, we have used a starting value $T=0.1$. (Obviously, very high values of $T$ would perturb all pixels of the FA input in such a way that it would be completely disturbed, whereas a relatively low initial $T$ may keep some features of the input while pushing it up from the relative minimum.) This will save a considerable amount of computing time. Plate $3(\mathrm{e})$ shows the reconstruction of the object shown in Plate 2(a), obtained in this way by using the speckle interferometry simulation data. The input to the SA was the FA reconstruction [Plate 3(a)]. A low-pass-filtered version of the reconstruction is shown in Plate 3(f). The quality of either Plate 3(e) or Plate 3(f) is not better than that of Plate 3(c) or Plate 3(d); however, the computing time is made much shorter by taking advantage of $\mathrm{FA}$ reconstruction; it is possible to reduce it almost by a factor of 3.

Plate 4(a) shows a picture of a quasar with a double nucleus, under study at present at the IAC, compressed to $32 \times 32$ pixels. Its autocorrelation and power spectrum are shown in Figs. 4(a) and 4(b), respectively, again represented by a logarithmic scale of gray levels. The FA reconstruction from noiseless data in the power spectrum is shown in Plate $4(\mathrm{~b})$; its error is $E=7.1 \times 10^{-2}$, and its cost function value is $F=7.8 \times 10^{-2}$; the values for the initial guess were $E_{0}=0.55$ and $F_{0}=261248$. By introducing this result as the input in the SA algorithm and starting it at $T=0.1$, we obtained the reconstruction shown in Plate $4(\mathrm{c})$; the error was $E=2.6 \times$ $10^{-3}$, and the cost function value was $F=8.5 \times 10^{-6}$. The improvement in the reconstruction obtained by using SA is remarkable.

Attempts were also made to introduce a SA reconstruction as the initial guess in a FA reconstruction. However, FA behaved as with a random input; namely, it improved the result only if it worked with a random input too, but it tended to stagnate otherwise. SA, on the other hand, improved the reconstructions when necessary, showing a remarkable ability to take the iterations out of a relative minimum. In fact, we also observed that a steady improvement was obtained by successive repetitions of the SA algorithm, each time starting at a moderately low temperature, say, $T=$ 0.1 .

Another possibility that was tested was as follows. The reconstruction was taken from a relative minimum by slightly raising $T$, say, to $T=0.1$, and then, instead of following with SA, a quenched version was applied, i.e., the value of $T$ was abruptly dropped to $T=0$. The following plates show this effect on a reconstruction given by FA. Plate 5(a) shows a FA reconstruction of the object shown in Plate 2(a); its error was $\mathrm{E} \simeq 8 \times 10^{-3}$. The pixels of this reconstruction are perturbed at $T=0.1$ until equilibrium is reached, and then a quenched SA $(T=0)$ process follows. This Monte Carlo procedure is repeated 10 times, each one starting with a slightly lower $T$. The result is shown in Plate 5(b); it has an error of $E \simeq 10^{-3}$ (once again the visual appearance is much better than the corresponding decay in $E$ ), and its resemblance to the original is excellent. A similar result would be obtained by applying 10 times the SA starting at moderately low $T$.

The above example shows that the SA algorithm can be exploited fully at the expense of increasing the computing time.

\section{CONCLUSIONS}

In this paper we have reported the possibilities of an improved version of the SA algorithm ${ }^{21}$ for solving the problem of phase retrieval. From our computer observations, we infer that SA requires a long computing time. It does not yield a quick spectacular reconstruction in a short time. However, it has the great advantage that it does not require the introduction of clever guesses or constraints; anyone with an average VAX computer or the equivalent at hand can use it. It seems to be flexible enough to permit the incorporation of a priori constraints. This flexibility makes it interesting for dealing with noisy data. Its behavior is, as far as we have observed, uniform, and the reconstruction may be steadily improved by increasing the steps of the algorithm or even by repeating it by increasing the $T$ parameter slightly to take the iteration out of a local minimum. It is also robust to noise. We believe that optimum use of the SA algorithm can be made by first taking advantage of the possibilities of FA, from the computing-time point of view. FA should be run first; then SA can improve the result when necessary. Its actual power in dealing with large arrays, we believe, is dependent on present-day computing costs.

\section{ACKNOWLEDGMENTS}

We thank D. Benitez for help with the speckle simulation data. This work has been supported by the Comision Asesora de Investigacion Científica y Técnica under grants 2502/83 and PA85/0239.

R. Navarro is also affiliated with Instituto de Astrofísica, de Canarias, 38071 La Laguna, Tenerife, Spain.

\section{REFERENCES}

1. Y. M. Bruck and L. G. Sodin, "On the ambiguity of the image reconstruction problem," Opt. Commun. 30, 304-308 (1979).

2. M. H. Hayes and J. H. McClellan, "Reducible polynomials in more than one variable," Proc. IEEE 70, 197-198 (1982).

3. J.R. Fienup, "Reconstruction of an object from the modulus of its Fourier transform," Opt. Lett. 3, 27-29 (1978).

4. J. R. Fienup, "Space object imaging through the turbulent atmosphere," Opt. Eng. 18, 529-534 (1979).

5. J. R. Fienup, "Phase retrieval algorithms: a comparison," Appl. Opt. 21, 2758-2769 (1982).

6. C. Y. C. Liu and A. W. Lohmann, "High resolution image formation through the turbulent atmosphere," Opt. Commun. 8, 372377 (1973). 
7. P. J. Napier and R. H. T. Bates, "Inferring phase information from modulus information in two-dimensional aperture synthesis," Astron. Astrophys. Suppl. 15, 427-430 (1974).

8. B. R. Frieden and D. G. Currie, "On unfolding the autocorrelation function," J. Opt. Soc. Am. 66, 1111 (A) (1976).

9. R. H. T. Bates, "Fourier phase problems are uniquely solvable in more than one dimension. I: Underlying theory," Optik 61, 247-262 (1982); K. L. Garden and R. H. T. Bates, "Fourier phase problems are uniquely solvable in more than one dimension. II: One-dimensional considerations," Optik 62, 131-142 (1982); W. R. Fright and R. M. T. Bates, "Fourier phase problems are uniquely solvable in more than one dimension. III: Computational examples for two dimensions," Optik 62, 219230 (1982).

10. H. H. Arsenault and K. Chalasinska-Macukov, "The solution to the phase retrieval problem using the sampling theorem," Opt. Commun. 47, 380-386 (1983); K. Chalasinska-Macukov and H. Arsenault, "Fast iterative solution to exact equations for the two-dimensional phase problem," J. Opt. Soc. Am. A 2, 46-50 (1985).

11. A. Levi and H. Stark, "Image restoration by the method of generalized projections with application to restoration from magnitude," J. Opt. Soc. Am. A 1, 932-943 (1984).

12. P. L. Van Hove, M. H. Hayes, J. S. Lim, and A. V. Oppenheim, "Signal reconstruction from Fourier transform magnitude," IEEE Trans. Acoust. Speech Signal Process. ASSP-31, 12861293 (1983).

13. H. V. Deighton, M. S. Scivier, and M. A. Fiddy, "Solution of the two-dimensional phase problem," Opt. Lett. 10, 250-251 (1985)

14. M. Nieto-Vesperinas, "A study on the performance of least square optimization methods in the problem of phase retrieval," Opt. Acta 33, 713-722 (1986).

15. J.R. Fienup and C. C. Wackerman, "Phase-retrieval stagnation problems and solutions," J. Opt. Soc. Am. A 3, 1897-1907 (1986).

16. J. C. Dainty and J. R. Fienup, "Phase retrieval and image recon- struction for astronomy," in Image Recovery: Theory and Application, H. Stark, ed. (Academic, New York, 1987), pp. 231275.

17. S. Kirkpatrick, C. D. Gelatt, and M. P. Vecchi, "Optimization by simulated annealing," Science 220, 671-680 (1983).

18. N. Metropolis, A. Rosenbluth, M. Rosenbluth, A. Teller, and E. Teller, "Equation of state calculations by fast computing machines," J. Chem. Phys. 21, 1087-1092 (1953).

19. W. E. Smith, H. H. Barrett, and R. G. Paxman, "Reconstruction of objects from coded images by simulated annealing," Opt. Lett. 8, 199-201 (1983); W. E. Smith, R. G. Paxman, and H. H. Barrett, "Image reconstruction from coded data: I. Reconstruction algorithms and experimental results," J. Opt. Soc. Am. A 2, 491-500 (1985).

20. H. H. Barrett, W. E. Smith, and R. G. Paxman, "Monte Carlo methods in optics," Acta Polytech. Scand. Appl. Phys. Ser. 149, 3 (1985).

21. M. Nieto-Vesperinas and J. A. Méndez, "Phase retrieval by Monte Carlo methods," Opt. Commun. 59, 249-254 (1986).

22. M. Pincus, "A closed form solution of certain programming problems," Oper. Res. 16, 690-694 (1968); "A Monte Carlo method for the approximate solution of certain types of constrained optimization problems," Oper. Res. 18, 1225-1228 (1970).

23. J. M. Hammersley and D. C. Handscomb, Monte Carlo Methods (Methuen, London, 1967), p. 113.

24. R. Y. Rubinstein, Simulation and the Monte Carlo Methods (Wiley, New York, 1981), p. 260.

25. F. J. Fuentes, M. Nieto-Vesperinas, and R. Navarro, "A FORTRAN program to obtain a function of two variables from its autocorrelation," submitted to Trans. Math. Software.

26. A. H. Greenaway, J. G. Walker, and J. A. G. Coombs, "Ambiguities in speckle reconstructions. Some ways of avoiding them," in Indirect Imaging, J. A. Roberts, ed. (Cambridge U. Press, Cambridge, 1984), pp. 111-117.

27. F. J. Fuentes, Instituto de Astrofísica de Canarias, La Laguna Tenerife, Spain (personal communication). 